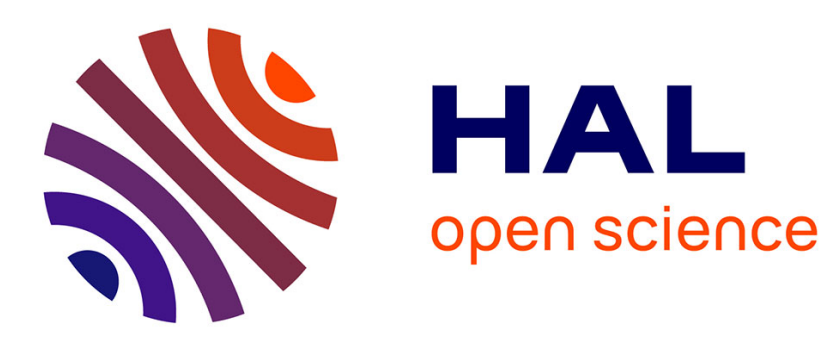

\title{
Real-time degradation monitoring and failure prediction of pulse energy conversion systems
}

Yury Kolokolov, Anna Monovskaya, Kondo Hloindo Adjallah

\section{To cite this version:}

Yury Kolokolov, Anna Monovskaya, Kondo Hloindo Adjallah. Real-time degradation monitoring and failure prediction of pulse energy conversion systems. Journal of Quality in Maintenance Engineering, 2007, 13 (2), pp.176-185. 10.1108/13552510710753078 . hal-03045174

\section{HAL Id: hal-03045174 \\ https://hal.univ-lorraine.fr/hal-03045174}

Submitted on 7 Dec 2020

HAL is a multi-disciplinary open access archive for the deposit and dissemination of scientific research documents, whether they are published or not. The documents may come from teaching and research institutions in France or abroad, or from public or private research centers.
L'archive ouverte pluridisciplinaire HAL, est destinée au dépôt et à la diffusion de documents scientifiques de niveau recherche, publiés ou non, émanant des établissements d'enseignement et de recherche français ou étrangers, des laboratoires publics ou privés. 


\title{
Real-time degradation monitoring and failure prediction of pulse energy conversion systems
}

\author{
Yury V. Kolokolov ${ }^{1}$, Anna V. Monovskaya ${ }^{1,2}$ \\ Kondo Hloindo Adjallah ${ }^{2}$ \\ ${ }^{1}$ State Technical University of Orel, Russia \\ Naugorskoje Shosse, 29, P.O. Box 2, 302020, Orel, Russia \\ Contact:\{anna.monovskaya;2kolo\}@mail.ru \\ ${ }^{2}$ Institut Charles Delaunay, University of Technology Troyes, \\ 12 rue Marie Curie, F.10010 Troyes Cedex, France \\ Contact:Kondo.adjallah@utt.fr
}

\begin{abstract}
Purpose - The paper presents a new approach for safe operation, and maintenance cost reduction, regarding electrical and electromechanical systems of power production, power conversion and power transmission, primarily in industrial units.

Design/methodology/approach - The paper adapts a theoretical approach to real-time monitoring of pulse energy conversion systems (PECSs), and prediction of abnormal dynamics incipient and developing failure. The approach utilizes the preliminary bifurcation analysis results and the geometrical interpretation of the fractal regularities in PECS dynamics, to reveal degradation development.

Findings - It turns out that this new approach enables to fill the joint requirements of real-time failure prediction of the high frequency power control devices, and of the relating failure symptoms to cause parameters. Discussions are led on the fundamental outcomes of numerical and experimental investigations of a DC-DC buck voltage converter with pulse-width-modulation (PWM) control.

Originality/value - About «failure prediction», we propose to pay attention, not to identification of the dynamics evolution specific reason, but real-time monitoring of this reason consequence incipient abnormal dynamics in the electrical and electromechanical systems -, that can lead to failure. The advantage of this approach consists in the possibility of taking into account PECS operating conditions of models ambiguity of both disturbing parameter changes and PECS behavior.

Practical implication - The real-time monitoring of incipient abnormal dynamics in key nonlinear devices of electrical and electromechanical systems constitutes a mean to predict and prevent failures. It provides invaluable information for deciding and planning predictive maintenance actions, from the insurance of optimal operating conditions until abnormal operating prevention, either by means of modification of controlled parameters and control laws, or in the worst case, by change of the power components' structure.
\end{abstract}

Keywords - Degradation monitoring, failure prediction, real-time, pulse energy conversion system, nonlinear dynamics, predictive maintenance.

Type of paper - Research paper 


\section{Introduction}

Pulse energy conversion systems (PECS) realize one of the promising power-saving technologies of power conversion and widely used in industry as one of the essential units of electrical and electromechanical systems of power production, conversion and transmission. So, the quality of PECS converted energy influences directly on the conditions in which the units connected with PECS will be force to operate and, correspondingly, on maintenance costs regarding practically each units of the system. So, enhancement of both efficiency and safety of electrical power pulse conversion processes can lead to achieve safety and reduction of maintenance cost on the electrical and electromechanical systems. Let consider more details this way.

While PECS designing, one proceeds from the requirement that an energy conversion process must be realized with a pulse-width modulation (PWM) frequency and retain the stable characteristics adjusted (hereafter "1-type process"). Contemporary PECS operate under actioning on their dynamics of a wide range of internal and external probable disturbances (for example, variation of an environmental temperature, PECS input energy and output load resistance, system degradation over time, availability of an initial technologic spread of PECS element values and so on). These operation conditions are not provided within the frame of PECS traditional design based on the small-signal analysis. Vice versa these attempts to enhance PECS dynamics robustness can lead to opposite effects (Kolokolov, 2005, c). So, while PECS is operating there are possibilities of 1-type process stability loss and appearance of stationary processes with another qualitative and quantitative characteristics: subharmonic (Iu, 2001), quasi-periodic (Aroudi, 2001), chaotic (di Bernardo, 2000).

These processes negative influence is appreciated from undesirable (leading to energy conversion process efficiency fall-off) until failure (leading to irreversible material damages and catastrophic environment pollutions - in the case of responsible technological processes). Let us consider one of the evident examples. Consisting of electric drive PECS dynamics must adapt to driven engine behavior (Busse et al. 1997). Failures occur if PECS operating process (1-type process) loses stability. Nikhil and Pecht (2006) presented a list of such failure modes and also they depicted failure precursor paradigms for their prevention. Methods of this problem solution should provide the necessary features for predictive maintenance decision making in terms of control strategy reconfiguration or of maintenance planning of the driven device. There exists, in frequency domain, many health monitoring and failure diagnosis methods for PWM drive systems. Except permanent fault with significant amplitude change which can be identified by some classical diagnosis methods (Richardeau et al. 2002), frequency domain methods based on Time-Frequency Transform and wavelet packet transform were developed to handle incipient degradation in PWM non stationary process (Yen and Lin, 2000, Zanardelli and

Strangas, 2005). To enhance effectiveness of those approaches, some predictive maintenance research specialists associated statistical classification and learning methods, but questions remain pertaining to their real-time implementation capability (Qiu et al. 2006, Awadallah and Morcos 2006). 
From the authors' point of view, 1-type process stability loss is the first characteristic sign of abnormal phenomena appearance before failure occurrence for all these examples of electrical and electromechanical energy conversion systems, and both efficient and safe operation of PECS depend on timely failure prediction. At the same time, existence of parameter variation ranges in which system behavior could not be treated uniquely (for example, the interspersing phenomenon in the case of stationary process existence domain overlapping in the parametrical space (Kolokolov, 2006), and flashing instability phenomenon about bifurcation boundaries (Kolokolov, $2005, c)$ is a peculiarity of PECS dynamics. Then, there is a unique possibility to forecast the 1-type process stability loss during a transitional process, duration of which is equal to fractions of a second in cause of the high-frequency modulation $\left(10^{-}\right.$ $\left.{ }^{3}-10^{-5} \mathrm{~s}\right)$. At that, the tendencies of semi-conductor component development are directed to improvement of energy conversion characteristics by commutation frequency increasing and, accordingly, to transitional process duration decreasing. So, hereafter «PECS failure prediction in real-time» means realization of 1-type process stability loss forecasting before a transitional process completion.

In that way, in the paper PECS operating is considered in the conditions of the model ambiguity of both disturbing parameter changes and PECS behavior. Thereupon, saying about «failure prediction», we propose to pay attention not to identification of the dynamics evolution specific reason but real-time monitoring of this reason consequence - abnormal dynamics incipience that leads to failure occurrence. The fundamental shortcoming of the known approaches regarding this problem solution is absence of the guarantee that a correct decision about the incipient stationary process is made before this process have settled. The developed approach (Kolokolov, $2005, b)$ presents an attempt to solve the one by means of the preliminary bifurcation analysis and geometrical interpretation of the fractal regularities revealed in PECS dynamics (Kolokolov, 2003, 2005,a). It allows projecting of the images of both preliminary and present data on PECS dynamics from multi-D parametrical and phase spaces into a special $2 \mathrm{D}$ space (2D stereo-projection). As the proposed idea is considered on the concrete example of DC-DC buck voltage converter with pulsewidth-modulation (PWM) control, then in section 2 an experimental plant is described and corresponding mathematical model are formed. Then, in section 3 the approach is presented and discussed by experimental results testifing their workability and effectiveness. Finally, in the last section, perspectives of the approach regarding predictive maintenance are indicated.

\section{Experimental plant}

An experimental plant of DC-DC buck voltage converter is considered in the paper (Fig. 1). This class of converter is widely used as low-voltage high-current compact power sources. In particular, the considered simplest variant of DC-DC buck voltage converter with proportional regulator represents a computer power source analogue. Nevertheless in dynamics of this regulator there are nonlinear phenomena which allow illustrating fundamental problems of PECS failure prediction. The experimental plant is made of four basic functional parts: power stage, regulator, IBM-PC with 
analog-digital converter board, auxiliary power supply. A power stage output voltage through a voltage sensor is transferred to the regulator where a reference voltage is subtracted from it, the obtained control error is transferred to a proportional unit. An amplification coefficient of the proportional unit corresponds to a digital code from LPT-port of IBM-PC. The control error amplified is transformed into a pulse pattern through a pulse-width modulator and transmitted to a driver. The driver forms voltage levels, which are necessary to control power stage transistors (S1 and S2). The transistors function in anti-phase mode and represent a synchronous rectifier. The power stage output voltage, through the voltage sensor, a throttle current, through a current sensor, and also an output voltage of the pulse-width modulator are transfered to the analog-digital converter board and transformed into 12-digit code.

Figure 1.

Functional scheme of the experimental plant (a), equivalent circuit of the synchronous buck voltage converter (b), appearance of the power stage (c)
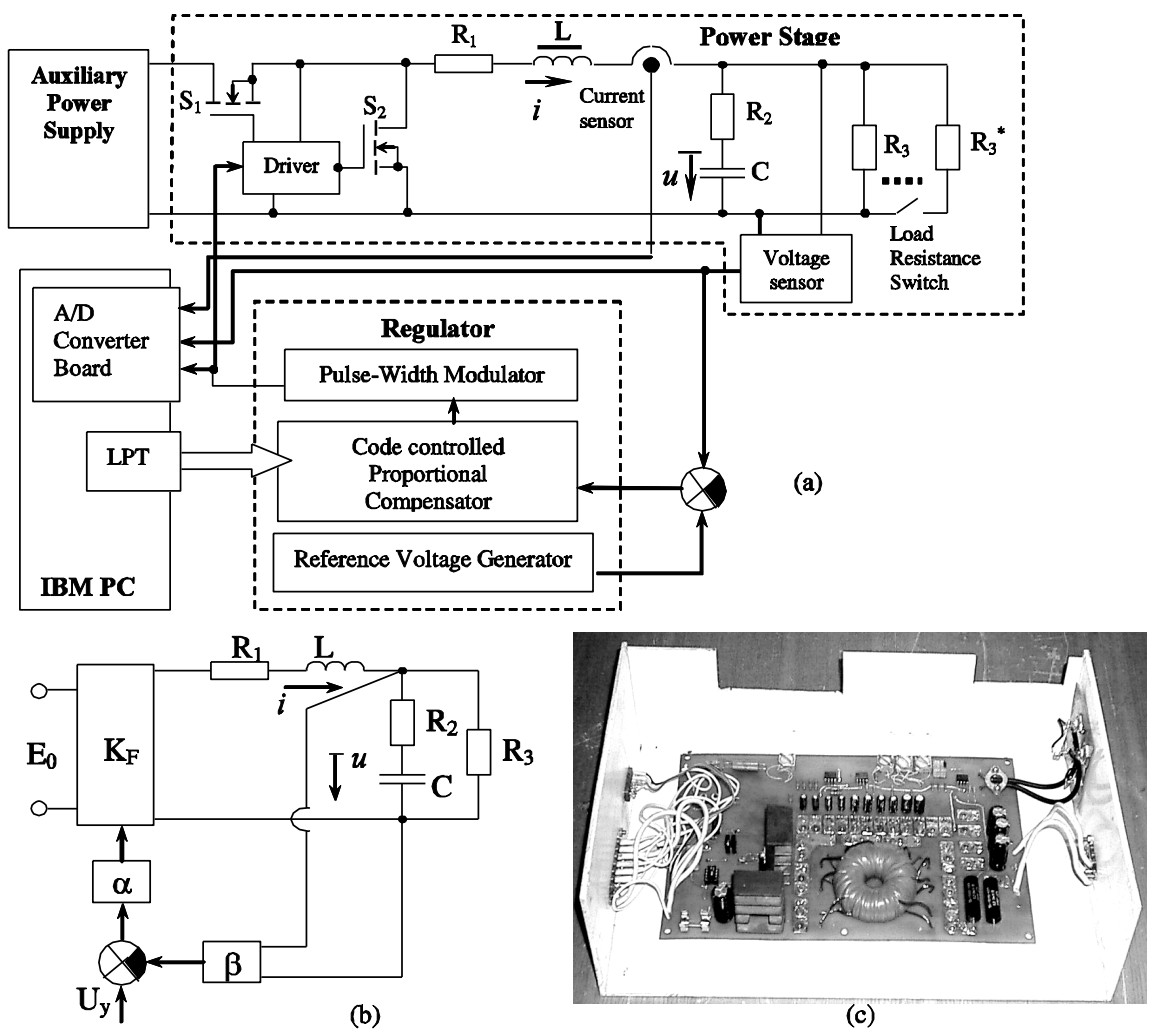

(c)

An equivalent circuit of the converter mathematical model is shown in Fig. 1b. The general form of the mathematical model is described by the differential equation system with a discontinuous right-hand side, which solutions for the constant structure sections are carried out similarly to the solution of the ordinary differential equation system:

$$
d X / d t=A X+B\left(K_{F}(\xi)\right),
$$


where $A$ is a matrix of invariable coefficients; $B$ is a matrix of the variable coefficients which describe the constant structure sections; $X$ is a system phase vector, including the throttle current $(i)$ and condenser voltage $(u) ; K_{F}$ is a pulse function, which is formed in accordance with the following algorithm:

$$
K_{F}=\left\{\begin{array}{l}
1, \quad \xi(t, X(t)) \geq 0 \\
0, \xi(t, X(t))<0
\end{array}\right.
$$

where $\xi(t, X(t))$ is a scalar commutation function of the phase vector $X$ at time $t$. Transitions through zero values of this function define the commutation moments when the system structure changes:

$$
\xi(t, X(t))=\alpha\left(U_{y}-\beta\left(R_{2} C \frac{d u}{d t}+u\right)-U_{0}\left(\frac{t}{T}-E_{1}\left(\frac{t}{T}\right)\right),\right.
$$

where $(k-1) T<t<k T, T$ is a period of the pulse-width modulation, $k=1,2, \ldots$ is a number of the periods, $E_{l}(\bullet)$ is an entire function, $\alpha, \beta, U_{y}, U_{o}$ are regulator parameters. The following mathematical model parameters were used in experimental investigations: $R_{I}=0,27 \Omega, R_{2}=0,18 \Omega, R_{3}=10 \ldots 50 \Omega, L=8,9 \cdot 10^{-4} \mathrm{H}, C=1,7 \cdot 10^{-4}$ $\mathrm{F}, E=24 \mathrm{~V}, \alpha=10 \ldots .50, \beta=1 / 4, U_{0}=2,6 \mathrm{~V}, U_{y}=3 \mathrm{~V}, f=1 / \mathrm{T}=8,61 \cdot 10^{3} \mathrm{~Hz}$.

Mathematical model solutions will be used in the following forms: time series $(i(t)$ and $u(t))$; phase trajectories $(u(i))$; shift mapping (Poincare mapping). The last one reflects a periodicity property of a stationary process (hereafter $m$-type process) and allows to differ stationary processes in the ratio of their unique frequency $\left(f_{m}=1 / m T\right)$ to the modulation one $f_{l}=1 / T(m=1)$. In this case fixed points of mapping $X_{j} f p$ are determined through the following condition:

$$
X_{1}^{f p}=G^{(1)}\left(X_{m}^{f p}\right)=G^{(m)}\left(X_{1}^{f p}\right),
$$

where $j=1,2 \ldots m, G^{(j)}-\mathrm{j}$-iteration of mapping. For example, two $m$-processes are used for discussed point illustration in the paper: 1-type process (operational process) with the frequency $f_{1}=1 / T$ and 10-type process (emergency) with the frequency $f_{10}=1 / 10 T$ and 2000001111 -symbolic characteristic, where $\gamma$ is a relative duration of the pulse. In the paper stationary and transitional process images are offered to form on the basis of such form solutions with usage of fractal regularities in PECS dynamics (Kolokolov, $2003 ; 2005$, a). The fractal regularities are considered from the geometrical point of view, and expressed the fact that a set of phase trajectories of one type stationary process, generated through the consecutive parameter variation, represents similar geometrical structures with certain shape (size and rotation) modifications. In particular a phase trajectory shape and its "sharp bends" map the unique consecution and duration of the time moments when a key element open or close (Fig. 2a,b) and a pulse function $\left(K_{F}\right)$ is equal to " 1 " or " 0 " correspondingly. So, hereafter let us accept 
that one of the fixed points of mapping corresponds to the time moment when $K_{F}$ value changes from « 4 » to «1» and has the minimum current value.
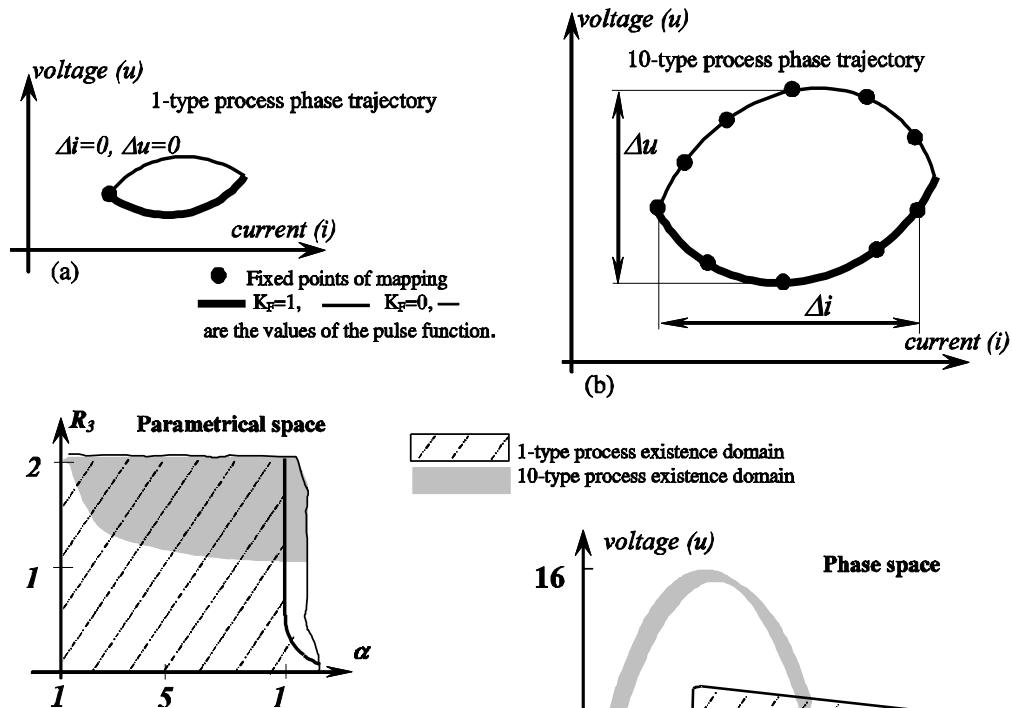

(b)

Figure 2.

Principles of phase trajectory transformation into $2 \mathrm{D}$ stereo-projection images by examples of 1,10 types processes (a),(b) and transitional process (f). Examples of 1,10- type process existence domains in the parametrical (c), phase (d) spaces and 2D stereo-projection (e), $\{\mathrm{g}\}$

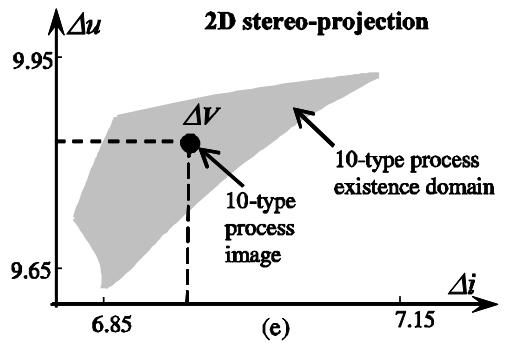

-type process existence domain

10-type process existence domain
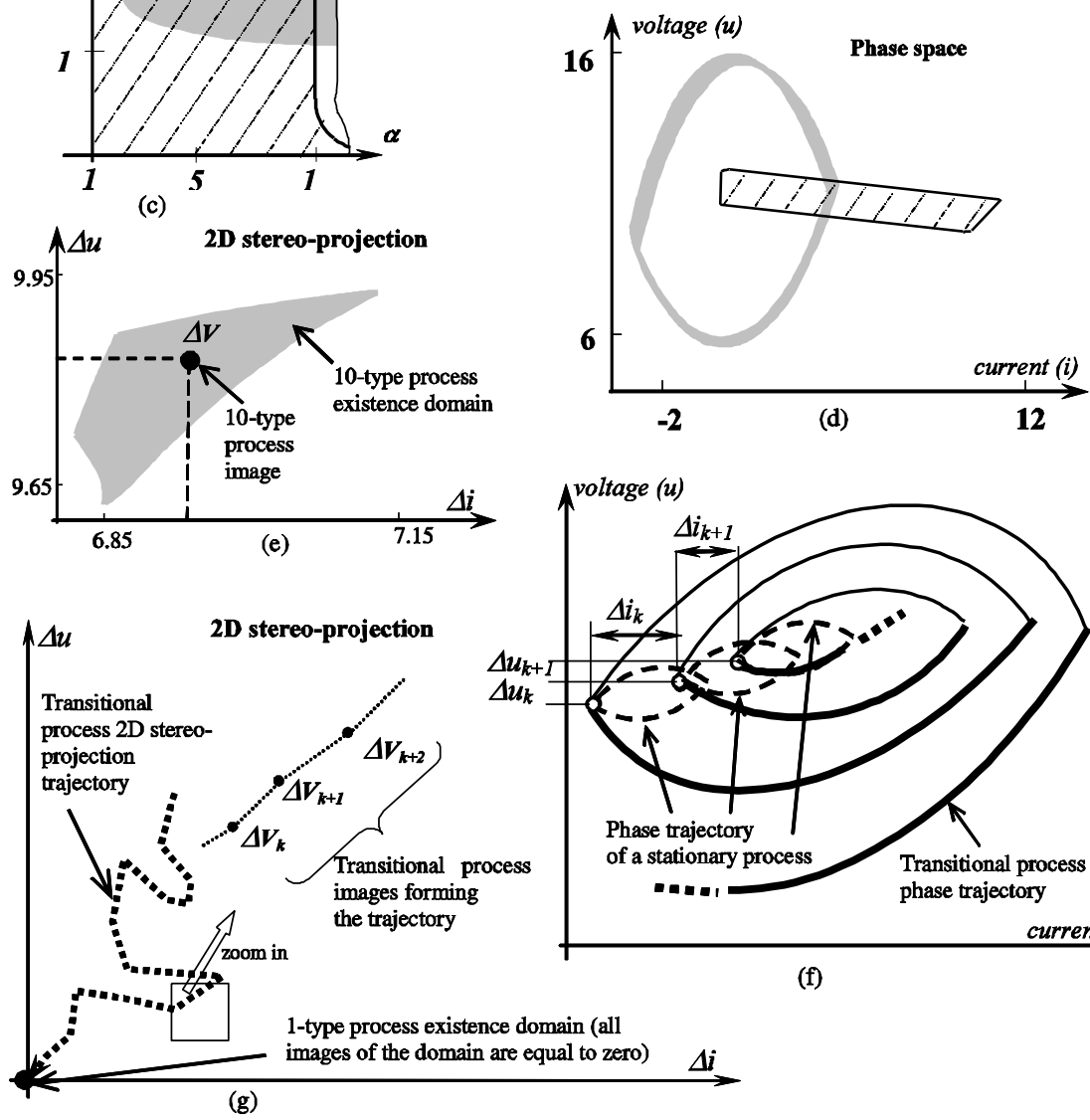


\section{Principles of the approach offered}

At present, the fundamental shortcoming in the most widespread approaches to nonlinear system identification and forecasting (for example, the traditional approaches (Ljung, 1999) - statistical, approximating based on Takens theorem usage, recurrent adaptive, and also the approaches actively developed - based on fuzzy logic and neuron-fuzzy techniques (Awadallah, 2004, 2006), neuronet technique (Calvo, 2004) and so on) is absence of the guarantee of real-time mode realization. Thereupon, it is necessary to mark the way concerned with usage of the bifurcation approach which gives the most effective means for visualization and analysis of nonlinear system dynamics. Following the preliminary bifurcation analysis, one can get a full pattern of nonlinear dynamics (i.e. a pattern of stationary process existence domains in the parameterical, phase or certain special spaces (Alpigini, 2000; Ionita, 2000)) and estimate the present system state regarding these domain bifurcation boundaries. So, the $1^{\text {st }}$ principle of PECS failure prediction in realtime supposes usage of preliminary data on PECS dynamics bifurcation analysis. But these data usage regarding PECS dynamics forecasting is connected with two key problems.

First, different type stationary process existence domains (for example, 1,10-type processes) can overlap in both parametrical (Fig. 2c) and phase (Fig. 2c) spaces, so there is multi-treatment correspondence between stationary process images and we can not efficiently use these preliminary data (Kolokolov, 2005, a). Moreover the problem is redoubled while space dimensionality increases (Kolokolov, 2005, b). Second, while real-time dynamics monitoring, the information on PECS dynamics in the form of time series is available but the present system process stationarity is not guaranteed. Correspondingly, since the parametrical diagram has not any information on transitional processes, one can analyze only time series fragments when a system process is stationary. Correspondingly, the necessity of solving these problem stipulates formation of the following principles of PECS failure prediction in realtime. The $2^{\mathrm{d}}$ one consists in a need to form $2 \mathrm{D}$ projection of the bifurcation diagram in which stationary process existence domains do not overlap. The $3^{\text {rd }}$ one consists in a need to ensure mapping of both stationary and transitional process images into the formed bifurcation diagram $2 \mathrm{D}$ projection.

One of the variants of these principles realization is the development of the approach to real-time forecasting of abnormal phenomana in PECS dynamics by means of usage of special 2D spaces (hereafter, 2D stereo-projections), which allow stereoprojecting of both preliminary and present data on PECS dynamics. At that, the preliminary data represent a projection of the stationary process existence domains of the parametrical (bifurcation) diagram, the present data - a projection of discontinuous time series of PECS phase variable instantaneous values. An image of the present stationary process is a point $\Delta V(\Delta i, \Delta u)$ within this process existence domain. An example of such image formation is illustrated in Fig.2 a,b for the abovementioned 1,10-types processes based on use of their phase trajectories: 


$$
\begin{gathered}
\Delta i=i_{\max }-i_{\min }, i_{\max }=\max \left\{i_{1}, i_{2}, i_{3}, \ldots i_{m}\right\}, i_{\min }=\min \left\{i_{1}, i_{2}, i_{3}, \ldots i_{m}\right\} \\
\Delta u=u_{\max }-u_{\min }, u_{\max }=\max \left\{u_{1}, u_{2}, u_{3}, \ldots u_{m}\right\}, u_{\min }=\min \left\{u_{1}, u_{2}, u_{3}, \ldots u_{m}\right\},
\end{gathered}
$$

where $\left(i_{j}, u_{j}\right)$ is position data of the fixed point of mapping $X_{j} f p, j=1,2, \ldots m$. The process existence domain made of these images can represents a point of origin (in the case of 1-type process domain, see Fig. 2g) and a polygon (in the case of 10-type process domain, see Fig. 2e). So, within 2D stereo-projection these domains do not overlap (as against the parametrical and phase spaces, Fig. 2c,d correspondingly). Interpretation(Fig. 2f) of a transitional process phase trajectory by analogy means that if the present process is stationary, then the fixed points of mapping will locate on the trajectory "sharp bends", and the corresponding image will be a point. While it is not happening, the present process is a transitional one and a set of points $\{\Delta V\}$ correspond to it. This set forms a trajectory - an uninterrupted broken line (Fig. 2g). Correspondingly, in the frame of the approach PECS dynamics is considered as alternation of stationary and transitional processes and mapped into 2D stereoprojection as a succession of the points (stationary process images) connected by the trajectories (transitional process images). In that way, a mechanism of stationary process type forecasting consists in that the present transitional process trajectory deviates from the existence domain of the stationary process losed stability and directs towards the existence domain of the stationary process settling in the system.

An estimation of the possibility of this mechanism practical realization was the purpose of the experimental investigations carried out on the experimental test bed presented in section 2. The investigations were planned starting from variation of two guided parameters - the load resistance and proportional unit amplification coefficient. Initial parameter variation ranges were determined starting from the results of preliminary numerical simulation of the mathematical model dynamics. Comparative analysis of the simulation and experimental results demonstrats realisability of the approach on the whole. The numerical simulation results was presented earlier in (Kolokolov, 2005, b). In this paper a characteristic example of the experimental investigation results is introduced (Fig. 3). The example illustrates that in case of probable negligible disturbances of both internal and external system parameters, experimental time series contain a "noise" which stipulates formation of a certain size neighborhood instead of one stationary process image (Fig. 3a). Nevertheless, one can quite clearly distinguish (Fig. 3b) both "grouping» about the stationary process existence domain boundaries (in the case of transitional process convergence towards this stationary process) and "going away» from the domain boundaries (in the case of transitional process beginning) the present transitional process trajectory images. So we, at least, can forecast normal (operating process and converging to the one transitional processes) and abnormal (everything else stationary processes and converging to the ones transitional processes) dynamics evolution. 


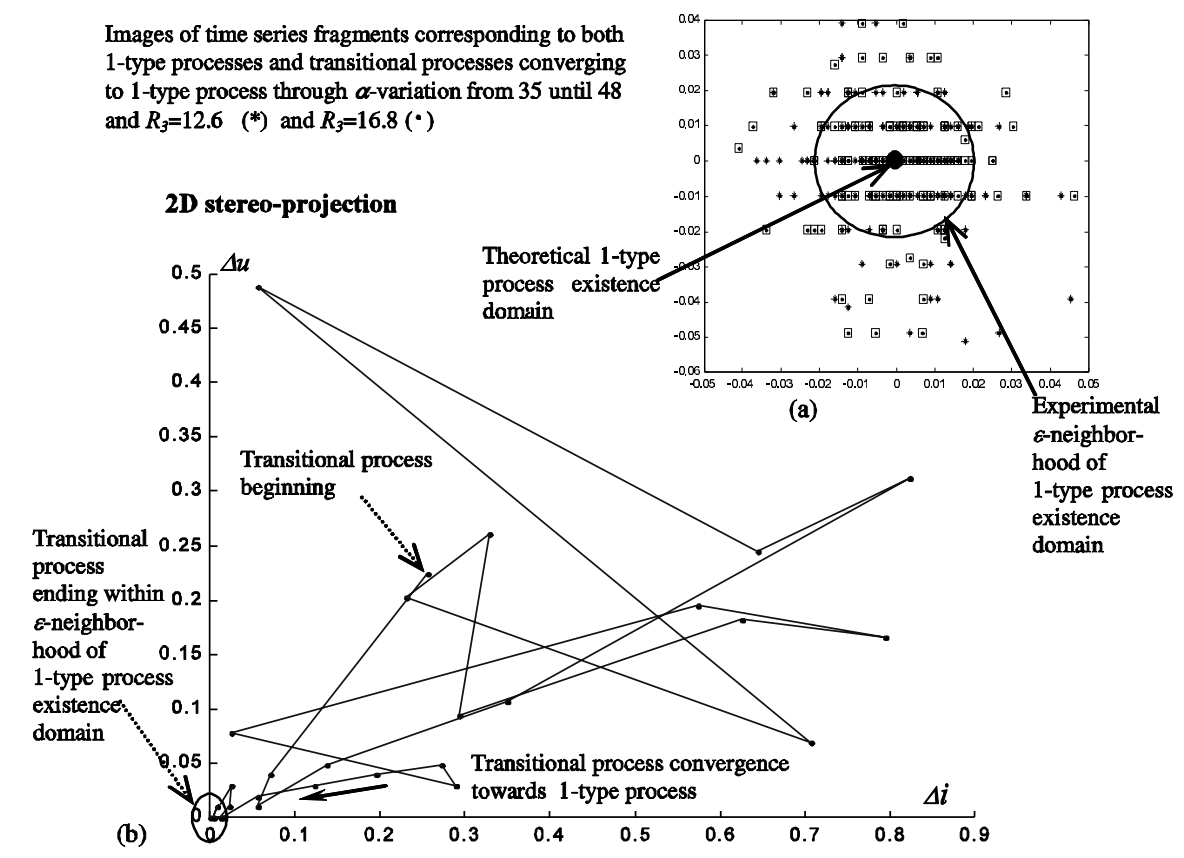

Figure 3.

\section{Conclusion}

In the paper, it is demonstrated how the theoretical approach to real-time PECS dynamics forecasting could be adapted to development of degradation monitoring strategy regarding failure prediction. This approach's advantage consists in the possibility of taking into account PECS operating conditions with models ambiguity of both disturbing parameter changes and PECS behavior. Three fundamental principles allowing realizing of PECS failure prediction in real-time were formulated:

1) Usage of preliminary data on PECS dynamics bifurcation analysis,

2) Forming of the bifurcation diagram $2 \mathrm{D}$ projection (2D stereo-projection) in which existence domains of stationary processes do not overlap,

3) Ensuring mapping of both stationary and transitional process images into the formed 2D stereo-projection.

From the practical point of view, it means that one can monitor, in real-time, incipient and developing abnormal dynamics allowing failure prediction. The approach provides invaluable information for deciding and planning predictive maintenance actions, from the insurance of optimal operating conditions until abnormal operating 
prevention, either by means of modification of controlled parameters and control laws, or in the worst case, by change of the power components' structure.

Experimental investigations of the approach were carried out on the experimental plant of DC-DC buck converter under variation of two parameters - the load resistance and proportional unit amplification coefficient. The satisfactory conformity of both simulation and experimental results enables to validate the implementation of this approach for PECS real-time failure prediction. In summary it is necessary to add that we used the example of the simplest PECS and realistic industrial devices that can be characterized by more complex dynamics.

\section{References}

Alpigini, J.J. (2000) "The evaluation and visualization of system performance in the chaotic dynamical systems", Information Sciences, Vol.127, pp. 173-192.

Aroudi, A.L., Leyva, R. (2001) "Quasi-periodic route to chaos in a PWM voltage-controlled dc-dc boost converter", IEEE Trans. on Circuits and Systems, Vol.48 No8, pp.967-978.

Awadallah, M. A. and Morcos M. M. (2006). Automatic Diagnosis and Location of OpenSwitch Fault in Brushless DC Motor Drives Using Wavelets and Neuro-Fuzzy Systems, IEEE Trans. on Energy Conversion, vol. 21, no. 1, pp. 104-111.

Awadallah, M.A., Morcos, M.M. (2004)"Switch fault diagnosis of PM brushless DC motor drive using adaptive fuzzy techniques", IEEE Trans. on Energy Conversion, Vol.19 No1, pp.226-227.

di Bernardo, M., Vasca, F. (2000) "Discrete-Time Maps for the Analysis of Bifurcations and Chaos in DC-DC Converters", IEEE Trans. on Circuits and Systems, Vol.47 No2, pp.130-142.

D. Busse, J. Erdman, R.J. Kerkman; Schlegel, D.; Skibinski, G. (1997), "Bearing current and their relationship to PWM drives", IEEE Trans. Power Electronics, vol. 12, no. 2, pp. 243-252.

Calvo, M., Malik,O.P. (2004) "Synchronous machine steady-state parameter estimation using neural networks", IEEE Trans. on Energy Conversion, Vol.19 No2, pp. 237-244.

Ionita, S. (2000) "A chaos theory perspective on system's failure", Information Sciences Vol.127, pp. 193-215.

Iu., H.H.C., Tse., C.K. (2001) "Bifurcation behavior in parallel-connected buck converters", IEEE Trans. on Circuits and Systems, Vol. 48 No2, pp.233-240.

Kolokolov, Yu.V., Monovskaya, A.V., Adjallah, K.H. (2003) "An identification of pulse system dynamics on the basis of fractal regularity use", A.L. Fradkov \& A.N. Churilov (Eds), 2003 IEEE Int. Conf. "Physics and Control" Proc. Aug. 20-23, 2003, StPetersburg, Russia, V.4, pp. 1184-1188.

Kolokolov Yu., Monovskaya A. (2005, a) "Fractal regularities of sub-harmonic motions perspective for pulse dynamics monitoring”, Chaos, Solitons \& Fractals, Vol.23, Issue 1, pp. 231-241

Kolokolov Yu., Monovskaya A. (2005, b) "Fractal principles of multidimensional data structurization for real-time pulse system dynamics forecasting and identification.", Chaos, Solitons \& Fractals 2005, Vol.25, Issue 5, pp. 991-1006.

Kolokolov, Yu., Koschinsky, S.L., Romanov, A.V. (2005, c) "Experimental research of the pulse system stability and robustnees", Proc. of the IEEE $3^{\mathrm{d}}$ Workshop "On Intelligent Data Acquisition and Advanced Computer Systems: Technology and Applications (IDAACS'2005)", 5-7 Sept., Sofia, Bulgaria, pp.624-627. 
Kolokolov, Yu.V., Koschinsky, S.L., Bagrov, V.V. (2006) "Experimental dynamics of electromechanical pulse energy conversion systems", IEEE Trans. on Instrumentation and Measurement, Vol.55 No1, pp. 35-43.

Ljung, L. (1999), System identification: Theory for the user, Prentice Hall, $2^{\text {nd }}$ edition.

Nikhil, M., Pecht M. G. (2006), Prognostics and Health Management of Electronics, IEEE Trans. Components and Packaging Technologies, vol. 29, no. 1, pp. 222-229.

Qiu, H., Lee, J., Lin J. (2006), Wavelet Filter-based Weak Signature Detection Method and its Application on Roller Bearing Prognostics, Journal of Sound and Vibration, Volume 289(4-5), pp. 1066-1090.

Richardeau, F., Baudesson P. Meynard T. A. (2002), Failures-Tolerance and Remedial Strategies of a PWM Multicell Inverter, IEEE Transactions on Power Electronics, vol. 17, no. 6 , pp. $905-912$.

Yen, G. G., Lin K-C. (2000), Wavelet Packet Feature Extraction for Vibration Monitoring, IEEE Transactions on Industrial Electronics, vol. 47, no. 3, pp. 650-666.

Zanardelli, W. G., Strangas E. G. (2005), Methods to Identify Intermittent Electrical and Mechanical Faults in Permanent Magnet AC Drives Based on Wavelet Analysis, IEEE Conference on Vehicle Power and Propulsion, Chicago, Illinois, USA, September 7-9, pp. 154-160. 